\title{
ESTRESSE OCUPACIONAL NA ASSISTÊNCIA DE CUIDADOS PALIATIVOS EM ONCOLOGIA*
}

\author{
Naira Agostini Rodrigues dos Santos ${ }^{1}$, Juliano dos Santos ${ }^{2}$, Vagnára Ribeiro da Silva ${ }^{3}$, Joanir Pereira Passos ${ }^{4}$
}

RESUMO: O objetivo deste estudo foi identificar o indicativo de estresse ocupacional em profissionais de enfermagem que atuam na assistência a pacientes com câncer em cuidados paliativos. Trata-se de um estudo descritivo, transversal, que contemplou 105 profissionais, enfermeiros e técnicos de enfermagem, de um hospital de cuidados paliativos oncológicos da cidade do Rio de Janeiro, Brasil. Para a coleta de dados, utilizouse um questionário com variáveis sociodemográficas e a Escala de Estresse no Trabalho. Essa etapa ocorreu no período de abril a julho de 2015. 58,1\% ( $n=61)$ dos profissionais apresentaram baixo nível de estresse ocupacional, $41 \%(n=43)$ deles relataram estresse moderado e $0,9 \%(n=u m)$ demonstrou alto nível de estresse. As variáveis relacionadas ao estresse foram: idade e tempo de formação profissional. Os dados sugerem que, apesar de estarem expostos a estressores como dor, sofrimento e morte, os profissionais estudados utilizam estratégias de enfrentamento eficazes na diminuição da percepção subjetiva do estresse.

DESCRITORES: Oncologia; Enfermagem oncológica; Cuidados paliativos; Estresse psicológico; Saúde do trabalhador.

\section{OCCUPATIONAL STRESS IN PALLIATIVE CARE IN ONCOLOGY}

ABSTRACT: The aim of the present study was to identify signs of occupational stress in nursing professionals that provide palliative care to patients with cancer. It is a descriptive and cross-sectional investigation that examined 105 nurses and nursing technicians from a hospital focused on palliative care in oncology in the city of Rio de Janeiro, Brazil. Data collection was carried out through the application of a questionnaire related to sociodemographic variables and the Work Stress Scale and took place from April to July 2015. Fifty-eight percent $(n=61)$ of the professionals presented a low level of occupational stress, $41 \%(n=43)$ reported a moderate intensity of stress and $0.9 \%$ or one participant declared to experience a high degree of stress. The variables related to stress were age and time of professional training. The outcomes suggest that, despite being exposed to stressful factors such as pain, suffering, and death, the studied professionals use effective coping strategies to reduce stress perception.

DESCRIPTORS: Medical oncology; Oncology nursing; Palliative care; Psychological stress; Occupational health.

\section{ESTRÉS LABORAL EN LA ATENCIÓN DE CUIDADOS PALIATIVOS EN ONCOLOGÍA}

RESUMEN: Se objetivó identificar el indicador de estrés laboral en profesionales de enfermería actuantes en atención de pacientes con cáncer en cuidados paliativos. Estudio descriptivo, transversal, incluyendo a 105 profesionales, enfermeros y auxiliares de enfermería, de hospital de cuidados paliativos oncológicos de Rio de Janeiro, Brasil. Datos recolectados mediante cuestionario con variables sociodemográficas y Escala de Estrés Laboral. Dicha etapa se realizó de abril a julio de 2015. El 58,1\% ( $n=61$ ) de los profesionales presentó bajo nivel de estrés laboral, $41 \%$ de ellos informó estrés moderado y $0,9 \%(n=1)$ expresó alto nivel de estrés. Las variables relacionadas al estrés fueron: edad y tiempo desde graduación profesional. Los datos sugieren que, a pesar de estar expuestos a estresores como dolor, sufrimiento y muerte, los profesionales estudiados utilizan estrategias de enfrentamiento eficaces para disminuir su percepción subjetiva del estrés.

DESCRIPTORES: Oncología; Enfermería Oncológica; Cuidados Paliativos; Estrés Psicológico; Salud Laboral.

\footnotetext{
*Artigo extraído da dissertação intitulada: "Estresse ocupacional e estratégias de enfrentamento da equipe de enfermagem: cuidados paliativos oncológicos". Universidade Federal do Estado do Rio de Janeiro, 2016.

${ }^{1}$ Enfermeira. Mestre em Enfermagem. Docente na Faculdade do Futuro. Manhuaçu, MG, Brasil.

2Enfermeiro. Doutor em Ciências. Enfermeiro do Hospital do Câncer III do Instituto Nacional de Câncer José de Alencar Gomes da Silva. Rio de Janeiro, RJ, Brasil.

${ }^{3}$ Enfermeira. Doutoranda em Enfermagem. Universidade Federal do Estado do Rio de Janeiro. Enfermeira do Hospital do Câncer I do Instituto Nacional de Câncer José de Alencar Gomes da Silva. Rio de Janeiro, RJ, Brasil.

${ }^{4}$ Enfermeira. Doutora em Enfermagem. Docente de Enfermagem da Universidade Federal do Estado do Rio de Janeiro. Rio de Janeiro, RJ, Brasil.
} 


\section{INTRODUÇÃO}

Ao longo da história da humanidade, o trabalho foi ganhando importância gradativa para indivíduos e organizações. Atualmente, desempenha papel central na vida das pessoas, constitui fonte de renda financeira, representa oportunidade de crescimento individual, identidade social e autoestima ${ }^{(1)}$.

No entanto, ao causar estresse, o trabalho pode repercutir negativamente nas condições de saúde do trabalhador, deixando-o exposto ao risco do estresse ocupacional. Assim, o profissional de enfermagem, pela natureza e característica de suas atividades, revela-se especialmente susceptível a esse fenômeno ${ }^{(1-2)}$.

O estresse ocupacional é definido como sendo um processo em que o indivíduo percebe demandas de trabalho no ambiente profissional como estressoras, que ao extrapolar suas capacidades de enfrentamento ou coping, provocam neste sujeito reações negativas ${ }^{(3)}$. Para que algo seja considerado estressor no ambiente de trabalho, é necessário que o indivíduo o perceba como tal $^{(4)}$.

Os profissionais de enfermagem que trabalham em serviço de oncologia estão expostos diariamente a situações geradoras de conflitos, tais como: as pressões impostas pelo modelo biomédico tradicional que prioriza a cura e a longevidade; as frequentes perdas por morte; o constante convívio que gera o vínculo com doentes graves, suas famílias e o luto desses familiares ${ }^{(5)}$.

Nesse contexto, destacam-se os profissionais de enfermagem que trabalham no cuidado paliativo. Esses trabalhadores lidam com prognósticos ruins; incurabilidade; manejo de sintomas que causam sofrimento e desconforto ao paciente, tais como: dor, sangramento, dispneia, constipação, náuseas, vômitos, fadiga e mutilações; além de estarem presentes no processo de morte e luto dos pacientes. Esses elementos concorrem para a penosidade do cotidiano dos profissionais de saúde, tornando-os vulneráveis para o acometimento do estresse ocupacional ${ }^{(6)}$.

Considerando esse cenário dotado de especificidades e de alta complexidade assistencial, gerencial e organizacional, a realização de estudos com a finalidade de explorar o fenômeno do estresse nos profissionais inseridos nesse modelo/proposta de cuidado é de grande importância, uma vez que fornece subsídios para intervenções relacionadas à saúde do trabalhador, com a elaboração de estratégias positivas, coletivas e individuais, a fim de minimizar os riscos ocupacionais e o estresse entre enfermeiros oncológicos que atuam nos cuidados paliativos ${ }^{(6)}$.

Diante do exposto, este estudo teve como objetivo identificar o indicativo de estresse ocupacional em profissionais de enfermagem que atuam na assistência a pacientes com câncer em cuidados paliativos.

\section{MÉTODO}

Estudo descritivo, transversal, que contemplou profissionais de enfermagem de uma unidade hospitalar pública especializada em cuidados paliativos oncológicos situada na cidade do Rio de Janeiro, Brasil. O local onde o estudo foi realizado é constituído por 56 leitos de internação, um ambulatório de atendimento multiprofissional, serviço de visita domiciliar e emergência. O quadro de enfermagem é constituído por 146 profissionais, sendo 48 enfermeiros e 98 técnicos de enfermagem.

Foram incluídos todos os profissionais de enfermagem que estavam inseridos na assistência direta a pacientes em um período superior a seis meses, englobando os turnos de diurno e noturno, independente da quantidade de vínculos empregatícios. Excluíram-se aqueles que estavam de férias ou de licença médica.

Dentre os 123 profissionais elegíveis para o estudo, todos foram abordados, dois se recusaram participar e 16 não devolveram os questionários respondidos nos prazos estabelecidos. Portanto, a amostra contemplou 105 profissionais, perfazendo 85,4\% da população alvo. Observou-se que, na amostra estudada, o tempo mínimo de atuação em cuidados paliativos foi de um ano.

O projeto de pesquisa foi aprovado pelos Comitês de Ética em Pesquisa das instituições envolvidas 
no estudo (UNIRIO - parecer 992.348 e INCA - parecer 1.012.056). A coleta de dados foi realizada entre abril e julho de 2015, de forma assistemática, no horário de funcionamento da unidade, com base na agenda e disponibilidade dos participantes da pesquisa.

Foram utilizados dois instrumentos para coleta de dados:

I) questionário sociodemográfico para caracterização dos participantes que continha as variáveis: sexo, idade, estado civil, categoria profissional, número de filhos, religião, tempo de formação profissional, tempo de atuação no cuidado paliativo oncológico, setor de atuação, turno de trabalho, outros vínculos empregatícios, tipo de vínculo empregatício nesta instituição e realização de atividades de lazer;

II) Escala de Estresse no Trabalho (EET), que avalia o estresse ocupacional por meio de indicadores de estresse organizacional de origem psicossocial. Trata-se de um instrumento unifatorial, de autorrelato, composto por 23 itens analisados por uma escala de concordância tipo Likert de cinco pontos, variando de "discordo totalmente" a "concordo totalmente", o qual foi construído e validado para trabalhadores de diferentes organizações e ocupações ${ }^{(7)}$.

O escore total da EET é a soma dos escores atribuídos a cada item e divididos pelo número total de itens da escala, obtendo-se a média aritmética. A partir da média, os participantes do estudo foram classificados quanto à intensidade de estresse. Tendo em vista que a escala oferece cinco opções de resposta, com valores variáveis de um a cinco, os pontos de corte adotados para o indicativo de estresse foram: baixo $(1,00$ a 2,00$)$, moderado $(2,01$ a 4,00$)$ ou alto $(4,01 \text { a } 5,00)^{(8)}$.

Para as análises bivariadas, a categoria moderado/alto estresse foi agrupada, já que apenas um profissional apresentou alto nível de estresse.

Para a análise descritiva, as variáveis qualitativas nominais ou ordinais foram descritas em números absolutos (n) e percentuais (\%), já as variáveis quantitativas foram descritas por meio de suas respectivas médias e desvios-padrão. A análise das relações entre a intensidade subjetiva do estresse e as variáveis nominais e ordinais foi realizada por meio do teste de Qui-quadrado de Pearson ou Razão de verossimilhança ou teste exato de Fischer, dependendo do número de casos por categoria ou quantidade de categorias da variável independente testada. Para as variáveis contínuas, foi utilizado o teste U Mann-Whitney ou teste $t$-Student, a depender da presença de normalidade das variáveis em análise.

As análises estatísticas foram realizadas utilizando o programa Statical Package for Social Science (SPSS) versão 20.0. Foram considerados estatisticamente significativos valores de $p<0,05$.

\section{RESUltados}

Dentre os profissionais de enfermagem estudados, houve predomínio do sexo feminino (82,9\%; $\mathrm{n}=87$ ) e a média de idade foi de 39,5 anos ( $D P=8,8)$. Os profissionais eram casados $(49,5 \% ; n=52)$, tinham, em média, um filho e referiram possuir alguma religião $(87,6 \%$; $=92)$, bem como realizar atividades de lazer $(91,4 \% ; n=96)$, conforme descrito na Tabela 1.

Tabela 1 - Características sociodemográficas dos profissionais de enfermagem que atuam na assistência a pacientes com câncer em cuidados paliativos. Rio de Janeiro, RJ, Brasil, 2015 (continua)

\begin{tabular}{|c|c|c|c|c|}
\hline Características sociodemográficas & $\begin{array}{c}\text { Baixo estresse } \\
\text { n (\%) }\end{array}$ & $\begin{array}{l}\text { Moderado/alto } \\
\text { estresse } n(\%)\end{array}$ & $\begin{array}{l}\text { Total } \\
\text { n }(\%) \\
\end{array}$ & Valor de $p$ \\
\hline \multicolumn{5}{|l|}{ Sexo } \\
\hline Feminino & $48(78,7)$ & $39(88,6)$ & $87(82,9)$ & \multirow[t]{2}{*}{$0,182^{*}$} \\
\hline Masculino & $13(21,3)$ & $5(11,4)$ & $18(17,1)$ & \\
\hline \multicolumn{5}{|l|}{ Idade (anos) } \\
\hline $20-40$ & $30(49,2)$ & $31(70,5)$ & $61(58,1)$ & \multirow[t]{2}{*}{ 0,029* } \\
\hline$>40$ & $31(50,8)$ & $13(29,5)$ & $44(41,9)$ & \\
\hline
\end{tabular}




\begin{tabular}{|c|c|c|c|c|}
\hline \multicolumn{5}{|l|}{ Estado civil } \\
\hline Solteiro & $16(26,2)$ & $15(34,1)$ & $31(29,5)$ & \multirow[t]{5}{*}{$0,834^{* *}$} \\
\hline Casado & $31(50,8)$ & $21(47,7)$ & $52(49,5)$ & \\
\hline Viúvo & $1(1,6)$ & $0(0)$ & $1(1)$ & \\
\hline Divorciado & $7(11,5)$ & $4(9,1)$ & $11(10,5)$ & \\
\hline União estável & $6(9,8)$ & $4(9,1)$ & $10(9,5)$ & \\
\hline \multicolumn{5}{|l|}{ Tem filhos } \\
\hline Sim & $42(68,9)$ & $24(54,5)$ & $66(62,9)$ & \multirow[t]{2}{*}{$0,134^{*}$} \\
\hline Não & $19(31,1)$ & $20(45,5)$ & $39(37,1)$ & \\
\hline \multicolumn{5}{|l|}{ Tem religião } \\
\hline Sim & $55(90,2)$ & $37(84,1)$ & $92(87,6)$ & \multirow[t]{2}{*}{$0,351^{*}$} \\
\hline Não & $6(9,8)$ & $7(15,9)$ & $13(12,4)$ & \\
\hline \multicolumn{5}{|c|}{ Atividades de lazer } \\
\hline Sim & $54(88,5)$ & $42(95,5)$ & $96(91,4)$ & \multirow[t]{2}{*}{$0,211 * * *$} \\
\hline Não & $7(11,5)$ & $2(4,5)$ & $9(8,6)$ & \\
\hline
\end{tabular}

*: teste qui-quadrado de Pearson **: razão de verossimilhança ***: teste exato de Fischer

No que se refere às características profissionais e relacionadas ao trabalho, $60 \%(n=63)$ era de técnicos de enfermagem; 36,2\% ( $n=38)$ tinha entre 11 e 20 anos de formação profissional e 35,2\% ( $n=37)$ dos profissionais tinham entre 1 e 10 anos de formação. Em relação ao tempo de atuação nos cuidados paliativos, 76,2\% $(n=80)$ referiu ter entre 1 a 10 anos de atuação, 62,9\% $(n=66)$ atuavam no setor de internação e $74,3 \%(n=78)$ era plantonista, sendo que 38,1\% ( $n=40)$ atuavam no plantão diurno e $36,2 \%$ $(n=38)$ exerciam suas funções no plantão noturno, conforme apresentado na Tabela 2.

Tabela 2 - Características profissionais e relacionadas ao trabalho dos profissionais de enfermagem que atuam na assistência a pacientes com câncer em cuidados paliativos. Rio de Janeiro, RJ, Brasil, 2015 (continua)

\begin{tabular}{|c|c|c|c|c|}
\hline $\begin{array}{l}\text { Características profissionais } \\
\text { relacionadas ao trabalho }\end{array}$ & $\begin{array}{l}\text { Baixo estresse } \\
\text { n (\%) }\end{array}$ & $\begin{array}{l}\text { Moderado/ Alto } \\
\text { estresse } n(\%)\end{array}$ & $\begin{array}{l}\text { Total } \\
\text { n }(\%)\end{array}$ & Valor de $p$ \\
\hline \multicolumn{5}{|l|}{ Categoria profissional } \\
\hline Enfermeiro & $21(34,4)$ & $21(47,7)$ & $42(40)$ & \multirow[t]{2}{*}{$0,170^{*}$} \\
\hline Técnico de enfermagem & $40(65,6)$ & $23(52,3)$ & $63(60)$ & \\
\hline \multicolumn{5}{|c|}{ Tempo de formação profissional } \\
\hline $1 \mathrm{a} 10$ anos & $16(26,2)$ & $21(47,7)$ & $37(35,2)$ & \multirow[t]{3}{*}{$0,043^{* *}$} \\
\hline 11 a 20 anos & $23(37,7)$ & $15(34,1)$ & $38(36,2)$ & \\
\hline$>20$ anos & $22(36,1)$ & $8(18,2)$ & $30(28,6)$ & \\
\hline \multicolumn{5}{|c|}{ Tempo de atuação em cuidados paliativos } \\
\hline 1 a 10 anos & $46(75,4)$ & $34(77,3)$ & $80(76,2)$ & \multirow[t]{3}{*}{$0,885^{* *}$} \\
\hline 11 a 20 anos & $13(21,3)$ & $8(18,2)$ & $21(20)$ & \\
\hline$>20$ anos & $2(3,3)$ & $2(4,5)$ & $4(3,8)$ & \\
\hline \multicolumn{5}{|l|}{ Setor de atuação } \\
\hline Internação & $40(65,6)$ & $26(59,1)$ & $66(62,9)$ & \multirow[t]{5}{*}{$0,689^{* *}$} \\
\hline Ambulatório & $4(6,6)$ & $2(4,5)$ & $6(5,7)$ & \\
\hline Visita domiciliar & $6(9,8)$ & $5(11,4)$ & $11(10,5)$ & \\
\hline Pronto atendimento & $10(16,4)$ & $8(18,2)$ & $18(17,1)$ & \\
\hline Posto avançado**** & $1(1,6)$ & $3(6,8)$ & $4(3,8)$ & \\
\hline
\end{tabular}




\begin{tabular}{|c|c|c|c|c|}
\hline \multicolumn{5}{|l|}{ Turno de trabalho } \\
\hline Plantonista diurno & $27(44,3)$ & $13(29,5)$ & $40(38,1)$ & \multirow[t]{3}{*}{$0,309^{* *}$} \\
\hline Plantonista noturno & $20(32,8)$ & $18(40,9)$ & $38(36,2)$ & \\
\hline Diarista***** & $14(23)$ & $13(29,5)$ & $27(25,7)$ & \\
\hline \multicolumn{5}{|c|}{ Outros vínculos empregatícios na Enfermagem } \\
\hline Sim & $26(42,6)$ & $15(34,1)$ & $41(39)$ & \multirow[t]{2}{*}{$0,377^{*}$} \\
\hline Não & $35(57,4)$ & $29(65,9)$ & $64(61)$ & \\
\hline \multicolumn{5}{|l|}{ Tipo de vínculo } \\
\hline Concursado estatutário & $45(73,8)$ & $38(86,4)$ & $83(79)$ & \multirow[t]{2}{*}{$0,118^{* *}$} \\
\hline Celetista & $16(26,2)$ & $6(13,6)$ & $22(21)$ & \\
\hline
\end{tabular}

*: teste qui-quadrado de Pearson

**: razão de verossimilhança

$* * *$ : teste exato de Fischer

****: setor considerado a porta de entrada do paciente para a unidade de cuidados paliativos onde trabalham apenas enfermeiros

$* * * * *$ : regime de trabalho de segunda a sexta-feira, excetuando feriados, com carga horária de 8 horas/dia

Os profissionais com baixo nível de estresse e nível de estresse moderado/alto foram diferentes em relação à idade $(p=0,029)$ e ao tempo de formação profissional $(p=0,043)$.

Quanto à idade, observou-se maior prevalência de estresse moderado/alto entre os profissionais com idade entre 20 e 40 anos em comparação àqueles com mais de 40 anos (70,5\% vs 29,5\%). Em relação ao tempo de formação profissional, verificou-se que os profissionais que possuíam entre 1 e 10 anos de formado apresentaram maior prevalência de estresse moderado/alto $(47,7 \% ; n=21)$, ao serem comparados àqueles profissionais com tempo de formação entre 11 e 20 anos $(34,1 \%$; $n=15)$ e mais de 20 anos de formação profissional oito (18,2\%), o que pôde ser verificado nas Tabelas 1 e 2 .

A maioria dos profissionais estudados apresentou estresse relacionado ao trabalho de intensidade baixa $58,1 \%(n=61)$, a prevalência de estresse moderado foi de $41 \%(n=43)$, e apenas um participante $(0,9 \%)$ apresentou alto nível de estresse, conforme mostrado na Tabela 3.

Tabela 3 - Prevalência de estresse dos profissionais de enfermagem que atuam na assistência a pacientes com câncer em cuidados paliativos, segundo a intensidade. Rio de Janeiro, RJ, Brasil, 2015

\begin{tabular}{lccc} 
Intervalo das médias & Intesidade & Frequência absoluta (n) & Frequência relativa (\%) \\
\hline 1,00 a 2,00 & Baixo estresse & 61 & 58.1 \\
\hline 2,01 a 4,00 & Moderado estresse & 43 & 41 \\
\hline 4,1 a 5,00 & Alto estresse & 1 & 0.9
\end{tabular}

As situações consideradas mais estressantes pelos participantes foram: tempo insuficiente para realizar as atividades de trabalho, ter que trabalhar durante muitas horas seguidas, e a forma de distribuição das tarefas. Já as situações consideradas menos estressantes foram: falta de confiança do superior sobre o trabalho, ter recebido ordens contraditórias, e a comunicação existente entre o profissional e o seu superior, de acordo com a descrição da Tabela 4. 
Tabela 4 - Distribuição das situações mais estressantes e das situações menos estressantes, segundo a percepção de profissionais de enfermagem que atuam na assistência a pacientes com câncer em cuidados paliativos. Rio de Janeiro, RJ, Brasil, 2015

\begin{tabular}{|c|c|c|}
\hline Situações mais estressantes & Média & DP \\
\hline O tempo insuficiente para realizar meu volume de trabalho deixa-me nervoso & 2,59 & 1,13 \\
\hline Fico de mau humor por ter que trabalhar durante muitas horas seguidas & 2,72 & 1,43 \\
\hline A forma como as tarefas são distribuídas em minha área tem me deixado nervoso & 2,74 & 1,35 \\
\hline \multicolumn{3}{|l|}{ Situações menos estressantes } \\
\hline $\begin{array}{l}\text { Tenho me sentido incomodado com a falta de confiança de meu superior sobre o meu } \\
\text { trabalho }\end{array}$ & 1,52 & 0,98 \\
\hline Tenho estado nervoso por meu superior me dar ordens contraditórias & 1,54 & 0,98 \\
\hline Sinto-me incomodado com a comunicação existente entre mim e meu superior & 1,67 & 1,02 \\
\hline
\end{tabular}

DP: Desvio Padrão

\section{- DISCUSSÃO}

Verificou-se o predomínio do sexo feminino, o que replica o perfil dos profissionais de enfermagem em âmbito nacional e mundial. Essa profissão tem o processo de atividade laboral influenciada pela história e cultura e reflete a relação existente entre a figura da mulher e a presença de habilidades e qualidades específicas que se enquadram nas atividades de cuidado ${ }^{(9)}$.

Alguns estudos trouxeram dados semelhantes quanto à faixa etária observada no presente estudo ${ }^{(8,10)}$ e constatou-se que, entre os profissionais pesquisados, quanto maior a idade, menor era a intensidade de estresse percebido. Pode-se pressupor que os profissionais com maior faixa etária são aqueles que apresentam possível maturidade e experiência, a qual é considerada como algo que possa contribuir nas decisões do trabalho e da vida individual ${ }^{(11)}$.

O maior tempo de formação e de atuação profissional confere maior domínio no campo de ação, segurança técnica e controle sobre as situações que surgem du rante a assistência de enfermagem ${ }^{(12)}$. Isto nos permite compreender que a vivência e a maturidade podem auxiliar na habilidade e na segurança para decidir e optar por estratégias de coping mais adequadas diante de situações estressantes, minimizando, desta maneira, a exposição e a percepção de estresse. Assim, no contexto deste estudo, entende-se que, ao longo do tempo de atuação, os profissionais tendem a criar estratégias mais eficazes a fim de conseguirem exercer suas funções com menos estresse laboral associado.

Infere-se que o tempo de atuação profissional no mesmo setor seja relevante, pois se torna mais fácil a interação com os demais profissionais e com o ambiente, podendo facilitar na percepção das situações desagradáveis e estressoras a fim de planejar situações para adaptação no ambiente de trabalho ${ }^{(8)}$. Observou-se, nesta pesquisa, que 75,4\% dos profissionais referiram ter entre 1 a 10 anos de atuação, $21,3 \%$ de 11 a 20 anos e 3,3\% mais de 20 anos no mesmo setor.

Em relação ao estado civil, houve predomínio de participantes casados, tal como verificado em estudos similares ${ }^{(8,12)}$. Quanto ao número de filhos, a baixa média observada reflete a baixa taxa de fecundidade apresentada nas últimas décadas no país, o que está relacionado ao êxodo rural ocorrido na década de 1970, com consequente aumento da inserção das mulheres no mercado de trabalho e nas instituições educacionais, bem como a utilização de métodos anticoncepcionais ${ }^{(13)}$.

Alguns autores consideram o companheiro e os filhos como sendo uma fortaleza, um suporte social importante para o profissional, representando segurança e apoio e facilitando ao enfrentamento do estresse ocupacional ${ }^{(10,14)}$.

Dentre os profissionais pesquisados, houve predomínio daqueles que referiram possuir uma religião e esse achado pode refletir o fato de algumas pessoas utilizarem sua crença como um apoio, 
tornando-a uma estratégia de coping, como observado entre enfermeiros oncológicos ${ }^{(15)}$. Essa mesma interpretação pode ser atribuída ao predomínio observado em relação à realização de atividades de lazer, já que tais ações podem funcionar para minimizar o desgaste emocional e físico gerado pelo trabalho ${ }^{(16)}$.

Há evidência da diferença dos níveis de estresse entre as categorias profissionais da enfermagem, apontando os enfermeiros com níveis de estresse mais alto do que os técnicos ${ }^{(12)}$, o que não foi comprovado neste estudo. Os altos níveis de estresse dos enfermeiros provavelmente estão associados com as atividades gerenciais e assistenciais que eles desenvolvem, como coordenação de equipes, unidades, atividade de supervisão e diversas cobranças da administração, além da atividade assistencial propriamente dita. Neste estudo, $60 \%$ dos profissionais consistiram de técnicos de enfermagem e $40 \%$ enfermeiros. Não houve diferença estatística significativa $(p=0,17)$ ao associar a categoria profissional com o nível de estresse.

No entanto, apesar da dinâmica de trabalho das diferentes categorias profissionais não ter sido objetivo deste estudo, pode-se inferir os resultados encontrados refletem a boa comunicação entre a equipe, a coerência nas deliberações e a confiança entre os diferentes membros, que foram as atividades relacionadas ao menor nível de estresse percebido.

Houve predomínio de profissionais que trabalhavam durante o dia (plantonistas diurnos e diaristas). Alguns estudos demonstram que o trabalho noturno altera o ritmo circadiano, podendo se relacionar com maior nível de estresse, comprometendo a saúde e auxiliando no envelhecimento precoce deste trabalhador ${ }^{(11)}$. No entanto, não foi encontrado associação entre o turno de trabalho e o nível de estresse, tal como observado em outro estudo que avaliou associação entre essas variáveis ${ }^{(12)}$.

É importante destacar que o turno de trabalho pode estar relacionado ao maior número de vínculos empregatícios dos profissionais sob o estudo. Apesar da dupla jornada de trabalho ser comum entre os profissionais de enfermagem ${ }^{(10)}$, no presente estudo houve predomínio de indivíduos com apenas um vínculo profissional. Esse dado pode estar relacionado ao fato de a maioria dos profissionais estudados serem funcionários públicos, com planos de carreira definidos, o que lhes confere estabilidade em relação ao vínculo empregatício e salários diferenciados, quando comparados aos profissionais da mesma categoria que atuam na iniciativa privada. Essa diferença salarial ocorre tanto em relação ao salário base, quanto sobre as gratificações por desempenho e titulação recebidas pelos profissionais que compuseram o presente estudo.

O trabalho executado por enfermeiros em unidades de saúde normalmente se caracteriza pelo contato com situações estressantes. Essas situações podem ser mais frequentes em especialidades cujos profissionais assistem pacientes com doenças crônico-degenerativas, como o câncer. Esses profissionais estão expostos em seu cotidiano de trabalho a situações geradoras de conflitos, como o convívio com pacientes com doença avançada, que apresentam limitações físicas, sociais, alguns com curativos extensos e odor desagradável, dor crônica, pacientes em fim de vida, e convívio com os familiares que estão em momento de perda.

Portanto, é razoável supor que esses trabalhadores estejam sujeitos a um alto nível de estresse. No entanto, essa hipótese não foi confirmada pelos achados deste estudo, no qual a maioria dos participantes apresentou baixo nível de estresse. Resultado semelhante foi observado entre profissionais de enfermagem que atuavam nos cuidados às mulheres com câncer de mama ${ }^{(17)}$.

Apesar de haver um predomínio de indivíduos com baixo nível de estresse, destaca-se que mais de $40 \%$ dos profissionais apresentaram nível de estresse moderado/alto. Esse resultado é relevante, já que os indivíduos com indicativo de estresse ocupacional moderado/alto podem apresentar sintomas físicos e emocionais relacionados à exaustão emocional e dependendo da intensidade e do tempo de exposição, podem até levar à morte ${ }^{(16)}$. Isso implica diretamente em prejuízo à saúde deste trabalhador e também para a instituição.

Os estressores que se destacaram estavam relacionados à sobrecarga de trabalho, à divisão do trabalho e à carga horária de trabalho. O mesmo resultado foi observado em uma pesquisa realizada com residentes médicos ${ }^{(16)}$. Cabe mencionar que a instituição na qual foi realizada a pesquisa estava passando por um período de transição de chefias geral e de enfermagem, o que pode ter influenciado na sobrecarga de trabalho aos profissionais, uma vez que a ausência de alguns profissionais no setor 
se fazia necessária para elaboração do plano de transição e de questões burocráticas.

Considerando as peculiaridades do paciente oncológico em cuidado paliativo, é notório o volume de trabalho para a equipe, ainda mais se considerarmos que raramente há um dimensionamento adequado do número de profissionais para assistência a esses pacientes, revelando a necessidade de maior preocupação pelos cuidados paliativos por parte dos gestores. Assim, a sobrecarga de trabalho é uma possível explicação para a elevada prevalência de estresse moderado/alto. Esse achado poderá vir a repercutir no aumento do absenteísmo e no prejuízo da saúde deste trabalhador, gerando desgaste psicológico, físico e social com consequente aumento do número de licenças médicas e pior dimensionamento de pessoal ${ }^{(18)}$.

Uma afirmativa entre as consideradas mais estressantes pelos investigados foi: "a forma como as tarefas são distribuídas em minha área tem me deixado nervoso". Nota-se que a divisão do trabalho foi considerada uma fonte de sofrimento, algo que os ameaça. A distribuição das tarefas norteia o trabalho, funcionando como um orientador da mente capaz de organizar e estabelecer a sequência para as ações. Organizando o trabalho, cada indivíduo avalia qual a melhor opção para a sua execução, direcionando-o para a satisfação no trabalho. Uma vez que isto não acontece, o trabalho poderá se transformar em um ameaçador do equilíbrio psicoemocional ${ }^{(16)}$. Tentar manter o ambiente laboral ameno, organizado e harmônico é um desafio para os gestores. A divisão de tarefas e as delegações de funções fazem parte da construção deste ambiente almejado ${ }^{(19)}$.

Cabe salientar que, para manter a saúde mental e física do profissional de enfermagem e evitar o seu adoecimento, é necessário identificar e interpretar a presença do evento estressor no ambiente de trabalho. Portanto, quanto maior a compreensão e o controle das situações e pressões no ambiente laboral, melhor será sua adaptação e, consequentemente, menor o indicativo de estresse ${ }^{(20)}$.

As situações relacionadas à menor intensidade de estresse mostram que a confiança entre o empregado e a chefia funciona como um fator protetor, em que o relacionamento e a comunicação ocorrem de maneira positiva entre os colegas de trabalho.

A comunicação é algo complexo que envolve interação, compartilhamento de mensagens, ideias, sentimentos e emoções, podendo influenciar o comportamento das outras pessoas que, por sua vez, reagirão de acordo com suas crenças, valores, história de vida e cultura. É um processo que exige interpretação, imaginação, sensibilidade, participação ativa, troca de energia. É um ato de compartilhamento a fim de estabelecer e manter relacionamentos com outras pessoas ${ }^{(21)}$.

As limitações deste estudo estão relacionadas ao seu delineamento, que não permite o estabelecimento de relações de causa e efeito entre os desfechos estudados e a amostra assistemática, que colabora para o viés de seleção. Recomenda-se novos estudos, com amostras sistemáticas, que contemplem outros centros de referência, na assistência a pacientes com câncer em cuidados paliativos e que utilizem uma metodologia que permita explorar o estresse entre os profissionais com baixa e moderada/alta intensidade do fenômeno.

\section{- CONSIDERAÇÕES FINAIS}

Os cuidados de enfermagem em cuidados paliativos oncológicos são de extrema importância, pois apresentam como foco o alívio dos sintomas físicos e psicológicos e tem como objetivo proporcionar qualidade de vida ao paciente, por meio de técnicas menos invasivas e tecnológicas. Esse tipo de trabalho faz com que os profissionais estejam expostos a extenuante carga emocional e física de trabalho, uma vez que o cuidado exige, além da técnica, atitudes de confortar, comunicar e acolher os pacientes e seus familiares neste momento de fragilidade da vida humana.

Sugere-se aos gestores de enfermagem estabeleçam mecanismos que contribuam no processo e nas condições de trabalho, diminuindo os riscos ocupacionais com vistas a minimizar os danos e os agravos à saúde do trabalhador e, consequentemente, proporcione melhoria da qualidade da assistência. 
1. Theme Filha MM, Costa MAS, Guilam MCR. Occupational stress and self-rated health among nurses. Rev. Latino-Am. Enfermagem. [Internet] 2013;21(2) [acesso em 15 nov 2016]. Disponível: http://dx.doi.org/10.1590/ S0104-11692013000200002.

2. Santos J, Meira KC, Pierin AMG. Prevalence of Cardiovascular Risk Factors in Brazilian Nursing Professionals: integrative literature review. J Hypertens. [Internet] 2014;32:e-140-140 [acesso em 20 jan 2017]. Disponível: http:// docplayer.net/35873903-Posters-session-s-a-t-u-r-d-a-y-p-o-s-t-e-r-s.html.

3. Paschoal T, Tamayo A. Impacto dos valores laborais e da interferência família: trabalho no estresse ocupacional. Psic.: Teor. e Pesq. [Internet] 2005;21(2) [acesso em 22 jul 2017]. Disponível: http://dx.doi.org/10.1590/S010237722005000200007.

4. Guido LA, Goulart CT, da Silva RM, Lopes LFD, Ferreira EM. Stress and Burnout among multidisciplinary residents. Rev. Latino-Am. Enfermagem. [Internet] 2012;20(6) [acesso em 20 jan 2015]. Disponível: http://dx.doi. org/10.1590/S0104-11692012000600008.

5. Eelen S, Bauwens S, Baillon C, Distelmans W, Jacobs E, Verzelen A. The prevalence of burnout among oncology professionals: oncologists are at risk of developing burnout, Psychooncology. [Internet] 2014;23(12) [acesso em 05 jul 2017] Disponível: http://dx.doi.org/10.1002/pon.3579.

6. dos Santos NAR, Gomes SV, Rodrigues CMA, dos Santos J, Passos JP. Estratégias de enfrentamento utilizadas pelos enfermeiros em cuidados paliativos oncológicos: revisão integrativa. Cogitare Enferm. [Internet] 2016;21(3) [acesso em 05 jul 2017]. Disponível: http://dx.doi.org/10.5380/ce.v21i3.45063.

7. Paschoal T, Tamayo A. Validação da Escala de Estresse no Trabalho. Estud. psicol. (Natal). [Internet] 2004;9(1) [acesso em 20 jan 2015]. Disponível: http://dx.doi.org/10.1590/S1413-294X2004000100006.

8. Stekel LMC. Estresse e coping entre auxiliares e técnicos de enfermagem de um hospital universitário [dissertação]. Santa Maria (RS): Universidade Federal de Santa Maria; 2011.

9. Elias EA, Souza IEO, Vieira LB. Significados do cuidado-de-si-mesmas de mulheres profissionais de enfermagem em uma unidade de pronto atendimento. Esc. Anna Nery. [Internet] 2014;18(3) [acesso em 20 jan 2015]. Disponível: http://dx.doi.org/10.5935/1414-8145.20140059.

10. Andolhe R, Guido LA, Linch GFC. Stress and oncological nursing: studies at Center of Researchs in Nursing. Online Braz J Nurs. [Internet] 2008;7(2) [acesso em 20 jan 2015]. Disponível: http://dx.doi.org/10.5935/16764285.20081505.

11. da Silva RM, Beck CLC, Guido LA, Lopes LFD, dos Santos JLG. Análise quantitativa da satisfação profissional dos enfermeiros que atuam no período noturno. Texto Contexto Enferm. [Internet] 2009;18(2) [acesso em 20 jan 2015]. Disponível: http://dx.doi.org/10.1590/S0104-07072009000200013.

12. Beneti ERR. Estresse e coping em trabalhadores de enfermagem de um hospital privado [dissertação]. Santa Maria (RS): Universidade Federal de Santa Maria; 2013.

13. Randa GMD, Mendes ACG, da Silva ALA. O envelhecimento populacional brasileiro: desafios e consequências sociais atuais e futuras. Rev. bras. geriatr. gerontol. [Internet] 2016;19(3) [acesso em 05 jul 2017]. Disponível: http:// dx.doi.org/10.1590/1809-98232016019.150140.

14. Rodrigues $A B$, Chaves EC. Stressing factors and coping strategies used by oncology nurses. Rev. LatinoAm Enfermagem. [Internet] 2008;16(1) [acesso em 20 jan 2015]. Disponível: http://dx.doi.org/10.1590/S010411692008000100004.

15. Ekedahl MA, Wengström Y. Caritas, spirituality and religiosity in nurses' coping. Eur J Cancer Care. [Internet] 2010;19(4) [acesso em 20 jan 2015]. Disponível: http://dx.doi.org/10.1111/j.1365-2354.2009.01089.x.

16. Bolzan MEO. Estresse, coping, burnout, sintomas depressivos e hardiness em residentes médicos [dissertação]. Santa Maria (RS): Universidade Federal de Santa Maria; 2012.

17. Linch GFC, Guido LA, Fantin SS. Nurses at a hemodynamics ward in Rio Grande do Sul, Brazil: profile and professional satisfaction. Texto Contexto Enferm. [Internet] 2010;19(3) [acesso em 20 jan 2015]. Disponível: http:// dx.doi.org/10.1590/S0104-07072010000300010. 
18. Martinato MCNB, Severo DF, Marchand EAA, de Siqueira HCH. Absenteísmo na enfermagem: uma revisão integrativa. Rev. Gaúcha Enferm. [Internet] 2010;31(1) [acesso em 20 jan 2015]. Disponível: http://dx.doi.org/10.1590/ S1983-14472010000100022.

19. Marques V, de Abreu JA. Estresse ocupacional, conceitos fundamentais para o seu gerenciamento. Associação Educacional Dom Bosco. [Internet] 2009 [acesso em 23 out 2015]. Disponível: http://www.aedb.br/seget/arquivos/ artigos09/288_Estresse_ocupacional,conceitosfundamentaisparaoseugerenciamento.pdf.

20. Negeliskii C, Lautert L. Occupational stress and Work Capacity of Nurses of a Hospital Group. Rev. LatinoAm. Enfermagem. [Internet] 2011;19(3) [acesso em 20 jan 2015]. Disponível: http://dx.doi.org/10.1590/S010411692011000300021.

21. Broca PV, Ferreira MA. A equipe de enfermagem e a comunicação não verbal. Reme, Rev. Min. Enferm.. [Internet] 2014;18(3) [acesso em 05 jul 2017]. Disponível: http://www.dx.doi.org/10.5935/1415-2762.20140051. 